\title{
TOPSIS Hybrid Multiattribute Group Decision-Making Based on Interval Pythagorean Fuzzy Numbers
}

\author{
H. U. Jun $(D)$, W. U. Junmin $(\mathbb{D}$, and W. U. Jie $\mathbb{B}$ \\ School of Economic and Management, Jiangsu University of Science and Technology, Zhenjiang, Jiangsu 212003, China \\ Correspondence should be addressed to H. U. Jun; 1434120126@qq.com
}

Received 18 October 2021; Revised 4 November 2021; Accepted 18 November 2021; Published 3 December 2021

Academic Editor: Massimiliano Ferrara

Copyright (c) $2021 \mathrm{H}$. U. Jun et al. This is an open access article distributed under the Creative Commons Attribution License, which permits unrestricted use, distribution, and reproduction in any medium, provided the original work is properly cited.

\begin{abstract}
Aiming at the mixed multiattribute group decision-making problem of interval Pythagorean fuzzy numbers, a weighted average (WA) operator model based on interval Pythagorean fuzzy sets is constructed. Furthermore, a decision-making method based on the technique for order preference by similarity to ideal solution (TOPSIS) method with interval Pythagorean fuzzy numbers is proposed. First, based on the completely unknown weights of decision-makers and attributes, interval Pythagorean fuzzy numbers are applied to TOPSIS group decision-making. Second, the interval Pythagorean fuzzy number WA operator is used to synthesize the evaluation matrices of multiple decision-makers into a comprehensive evaluation matrix, and the relative closeness of each scheme is calculated based on the TOPSIS decision-making method. Finally, an example is given to illustrate the rationality and effectiveness of the proposed method.
\end{abstract}

\section{Introduction}

In a real decision-making process, the information obtained by decision-makers is often fuzzy and uncertain. Therefore, decision-making research based on fuzzy information is essential. Zadeh [1] first proposed the concept of a fuzzy set in 1965. Experts and scholars began to study fuzzy decision-making, and based on this, they carried out a series of studies, examining topics such as intuitionistic fuzzy sets [2-10] and interval fuzzy sets [11-16]. In 2013, Yager [3] first proposed the concept of a Pythagorean fuzzy number based on intuitionistic fuzzy sets. Experts and scholars began to apply Pythagorean fuzzy numbers to various decisions. Peng [4] defined the similarity and distance measures of Pythagorean fuzzy numbers. Chen [5] extended the Pythagorean fuzzy environment to the VIKOR decision model. Ren et al. [6] combined a Pythagorean fuzzy set with the TODIM method. Garg [7], Liu Weifeng [8], and Li Peng $[9,10]$ applied Pythagorean fuzzy numbers to geometric clustering operators, ordered weighting operators, generalized WOWA operators, and other operators.

In the existing decision-making processes, it is often difficult for decision-makers to use an accurate value to evaluate the advantages and disadvantages of the scheme.
Methods using interval Pythagorean fuzzy values are more conducive to expressing fuzzy information. Peng [11] and Muhammad [12] defined interval Pythagorean fuzzy integration operators. Zhang [13], Li $\mathrm{Na}[14,15]$, and Lin Wenhao [16] applied an interval Pythagorean fuzzy environment to decision-making processes, such as the hierarchical qualitative flexible multiple criteria method (QUALIFLEX), AQM method, and VIKOR operator.

The technique for order preference by similarity to ideal solution (TOPSIS) decision-making method is a widely used multiattribute decision-making method, and it is one the most frequently used techniques to deal with multicriteria group decision-making (MCGDM) conflicts. Umer et al. [17] expanded the TOPSIS method using a distance method with interval type-2 trapezoidal Pythagorean fuzzy numbers (IT2TrPFNs) and applied it for MCGDM dilemmas by considering the attitudes and perspectives of the decisionmakers. Pishyar et al. [18] used the TOPSIS and analytic hierarchy process (AHP) methods to determine, prioritize, and assess the most effective desertification indices. Kacprzak et al. [19] presented a new approach for ranking the alternatives for group decision-making using the TOPSIS method based on ordered fuzzy numbers. Rezaei 
et al. [20] developed a method to solve the sustainable circular partner selection problem with completely unknown decision-making experts. Du Yingxue et al. [21] proposed a triangular Pythagorean fuzzy TOPSIS decisionmaking method. Qu Guohua [22] proposed an intuitionistic fuzziness $\lambda$-Shapley Choquet integral operator TOPSIS multiattribute group decision-making method. Zhao and others [23] proposed a new TOPSIS decision-making method based on intuitionistic fuzzy sets, interval fuzzy sets, and other evaluation information.

In recent years, experts and scholars have conducted multidomain, in-depth research in the field of Pythagorean fuzzy numbers, extended Pythagorean fuzzy numbers to interval Pythagorean fuzzy numbers, and studied various operators and decision models in the environment of interval Pythagorean fuzzy numbers. However, the application of the TOPSIS decision method in an interval Pythagorean fuzzy environment is rare. Therefore, the weighted average (WA) operator of interval Pythagorean fuzzy numbers is defined, and a TOPSIS hybrid multiattribute decisionmaking method based on interval Pythagorean fuzzy numbers is presented. The advantages of this method are as follows: (1) it alleviates the loss of information in the decision-making process, (2) it effectively solves different types of attribute information problems and fully utilizes the attribute advantages of each decision-maker, and (3) it enlarges the gap between the closeness of each scheme and the advantages and disadvantages, making the decision-making results more convincing.

\section{Basic Concepts}

\subsection{Pythagorean Fuzzy Sets}

Definition 1 (see [1]). Suppose $X$ is a given universe and $P=\left\{\left\langle x, P\left(\mu_{P}(x), v_{P}(x)\right)\right\rangle \mid x \in X\right\}$ is a Pythagorean fuzzy set on $X . \mu_{P}(x)$ represents the membership degree of $x$ belonging to $P$, and $\nu_{P}(x)$ indicates that $x$ belongs to the nonmembership degree of $P . \mu_{P}(x)$ and $\nu_{P}(x)$ satisfy $0 \leq \mu_{P}(x), \nu_{P}(x) \leq 1$, and $0 \leq \mu_{P}^{2}(x)+\nu_{P}^{2}(x) \leq 1$. The hesitation degree of $x$ to $P$ is defined as $\pi_{P}(x)=$ $\sqrt{1-\left(\mu_{P}^{2}(x)+\nu_{P}^{2}(x)\right)}$. For simplicity, $\left(\mu_{P}(x), \nu_{P}(x)\right)$ is called a Pythagorean fuzzy number, and it is abbreviated as $\alpha=\left(\mu_{P}(x), \nu_{P}(x)\right)$ in this paper.

Definition 2 (see [6]). For the Pythagorean fuzzy number $\alpha=\left(\mu_{P}(x), v_{P}(x)\right)$, the scoring function of $\alpha$ is defined as $S(\alpha)=\mu_{P}^{2}(x)-\nu_{P}^{2}(x)$ and the precision function $\alpha$ is defined as $H(\alpha)=\mu_{P}^{2}(x)+\nu_{P}^{2}(x)$, where $S(\alpha) \in[-1,1]$ and $H(\alpha) \in[0,1]$.

Definition 3 (see [6]). For two Pythagorean fuzzy numbers $\alpha_{i}=P\left(\mu_{P i}(x), v_{P i}(x)\right)(i=1,2)$, the comparison is as follows:

(1) Suppose $S\left(\alpha_{1}\right)=S\left(\alpha_{2}\right)$. If $H\left(\alpha_{1}\right)=H\left(\alpha_{2}\right)$, then $\alpha_{1}=\alpha_{2}$, and if $H\left(\alpha_{1}\right)<H\left(\alpha_{2}\right)$, then $\alpha_{1}<\alpha_{2}$.

(2) Suppose $S\left(\alpha_{1}\right)>S\left(\alpha_{2}\right)$. Then, $\alpha_{1}>\alpha_{2}$.
Definition 4 (see [6]). For two Pythagorean fuzzy numbers $\alpha_{1}=P\left(\mu_{P 1}(x), \nu_{P 1}(x)\right)$ and $\alpha_{2}=P\left(\mu_{P 2}(x), \nu_{P 2}(x)\right)$, the following operation rules are satisfied:

$$
\begin{aligned}
& \text { (1) } \alpha_{1} \oplus \alpha_{2}=\left(\sqrt{\mu_{P 1}^{2}(x)+\mu_{P 2}^{2}(x)-\mu_{P 1}^{2}(x) \mu_{P 2}^{2}(x)},\right. \\
& \left.v_{P 1}(x) v_{P 2}(x)\right) \\
& \text { (2) } \alpha_{1} \otimes \alpha_{2}= \\
& \text { ( } \left.\mu_{P 1}(x) \mu_{P 2}(x), \sqrt{v_{P 1}^{2}(x)+v_{P 2}^{2}(x)-v_{P 1}^{2}(x) \nu_{P 2}^{2}(x)}\right) \\
& \text { (3) } \lambda \alpha=\left(1-\left(1-\mu_{P}^{2}(x)\right)^{\lambda}, v_{P}^{\lambda}(x)\right) \\
& \text { (4) } \alpha^{\lambda}=\left(\mu_{P}^{\lambda}(x), 1-\left(1-v_{P}^{2}(x)\right)^{\lambda}\right)
\end{aligned}
$$

2.2. Interval Pythagorean Fuzzy Sets. In decision-making, scholars have proposed the concept and algorithms of interval Pythagorean fuzzy numbers. An interval Pythagorean fuzzy number adds the upper and lower limits of the membership and nonmembership based on the Pythagorean fuzzy number.

Definition 5 (see [13]). Suppose $X$ is a given universe and $\widetilde{P}=\left\{\left\langle x, \widetilde{P}\left(\mu_{\vec{P}}^{-}(x), \mu_{P}^{ \pm}(x)\right), \widetilde{P}\left(\nu_{\vec{P}}^{-}(x), \nu_{P}^{ \pm}(x)\right)\right\rangle \mid x \in X\right\}$ is an interval Pythagorean fuzzy set on $X . \mu_{P}^{-}(x)$ and $\mu_{P}^{+}(x)$ are the lower and upper bounds of the membership degree, respectively, and $\nu_{\widetilde{P}}^{-}(x)$ and $\nu_{\widetilde{P}}^{+}(x)$ are the lower and upper bounds of the nonmembership degree, respectively. The interval Pythagorean fuzzy set $\widetilde{P}$ satisfies $0 \leq\left(\mu_{P}^{+}(x)\right)^{2}+\left(\nu_{\widetilde{P}}^{-}(x)\right)^{2} \leq 1$. The hesitation degree of $x$ to the interval Pythagorean fuzzy set $\widetilde{P}$ is defined as $\pi_{\widetilde{P}}(x)=\left[\pi_{\widetilde{P}}^{-}(x), \pi_{\widetilde{P}}^{ \pm}(x)\right]=\left[\sqrt{1-\left(\mu_{\tilde{P}}^{ \pm}(x)\right)^{2}-\left(\nu_{\tilde{P}}^{ \pm}(x)\right)^{2}}\right.$, $\left.\sqrt{1-\left(\mu_{P}^{-}(x)\right)^{2}-\left(\nu_{\tilde{P}}^{-}(x)\right)^{2}}\right]$.

Definition 6 (see [13]). For the interval Pythagorean fuzzy number $\widetilde{\alpha}=\left(\widetilde{P}\left(\mu_{P}^{-}(x), \mu_{\widetilde{P}}^{ \pm}(x)\right), \widetilde{P}\left(\nu_{\widetilde{P}}^{-}(x), \nu_{\widetilde{P}}^{ \pm}(x)\right)\right.$, the scoring function $\alpha$ is defined as $S(\widetilde{\alpha})=1 / 2\left(\left(\mu_{\tilde{P}}^{-}(x)\right)^{2}-\left(\nu_{\widetilde{P}}^{-}(x)\right)^{2}+\right.$ $\left.\left(\mu_{P}^{ \pm}(x)\right)^{2}-\left(\nu_{P}^{ \pm}(x)\right)^{2}\right)$ and the precision function $\alpha$ is defined as $H(\widetilde{\alpha})=1 / 2\left(\left(\mu_{\widetilde{P}}^{-}(x)\right)^{2}+\left(\nu_{\widetilde{P}}^{-}(x)\right)^{2}+\left(\mu_{P}^{+}(x)\right)^{2}+\left(\nu_{\widetilde{P}}^{+}(x)\right)^{2}\right)$, where $S(\widetilde{\alpha}) \in[-1,1]$ and $H(\widetilde{\alpha}) \in[0,1]$.

Definition 7 (see [13]). For two interval Pythagorean fuzzy numbers $\quad \widetilde{\alpha}_{i}=\left(\widetilde{P}\left(\mu_{\widetilde{P} i}^{-}(x), \mu_{\widetilde{P} i}^{+}(x)\right), \widetilde{P}\left(\nu_{\widetilde{P} i}^{-}(x), \nu_{\widetilde{P} i}^{+}(x)\right)\right)(i=$ $1,2)$, the comparison is as follows:

(1) Suppose $S\left(\widetilde{\alpha}_{1}\right)=S\left(\widetilde{\alpha}_{2}\right)$. If $H\left(\widetilde{\alpha}_{1}\right)=H\left(\widetilde{\alpha}_{2}\right)$, then $\widetilde{\alpha}_{1}=\widetilde{\alpha}_{2}$, and if $H\left(\widetilde{\alpha}_{1}\right)<H\left(\widetilde{\alpha}_{2}\right)$, then $\widetilde{\alpha}_{1}<\widetilde{\alpha}_{2}$

(2) Suppose $S\left(\widetilde{\alpha}_{1}\right)>S\left(\widetilde{\alpha}_{2}\right)$. Then, $\widetilde{\alpha}_{1}>\widetilde{\alpha}_{2}$

Definition 8 (see [13]). For two interval Pythagorean fuzzy numbers $\quad \widetilde{\alpha}_{i}=\left(\widetilde{P}\left(\mu_{\widetilde{P} i}^{-}(x), \mu_{\widetilde{P} i}^{+}(x)\right), \widetilde{P}\left(\nu_{\widetilde{P} i}^{-}(x), \nu_{\widetilde{P} i}^{+}(x)\right)\right)(i=$ $1,2)$, the following operations are satisfied:

$$
\begin{aligned}
& \text { (1) } \widetilde{\alpha}_{1} \oplus \widetilde{\alpha}_{2}=\left(\left[\sqrt{\left(\mu_{P 1}^{\bar{\tau}}(x)\right)^{2}+\left(\mu_{P 2}^{\bar{\tau}}(x)\right)^{2}-\left(\mu_{P_{1}}^{\bar{\tau}}(x)\right)^{2}\left(\mu_{P 2}^{\bar{\tau}}\right.}\right.\right. \\
& (x))^{2}, \quad \sqrt{\left(\mu_{P 1}^{ \pm}(x)\right)^{2}+\left(\mu_{P 2}^{ \pm}(x)\right)^{2}} \quad-\left(\mu_{P 1}^{ \pm}(x)\right)^{2}\left(\mu_{P 2}^{ \pm}\right. \\
& \left.\left.(x))^{2}\right],\left[\nu_{P}^{-}(x) \nu_{P 2}^{-}(x), \nu_{P 1}^{ \pm}(x) \nu_{P 2}^{ \pm}(x)\right]\right)
\end{aligned}
$$


(2) $\tilde{\alpha}_{1} \otimes \widetilde{\alpha}_{2}=\left(\left[\mu \bar{\tau}_{P 1}(x) \mu \bar{\tau}_{P 2}(x), \mu_{P 1}^{ \pm}(x) \mu_{P 2}^{ \pm}(x)\right],\left[\sqrt{\left(\nu_{P 1}^{\bar{\tau}}\right.}\right.\right.$ $(x))^{2}+\left(\nu_{P 2}^{\bar{\tau}}(x)\right)^{2}-\left(\nu_{P 1}^{\overline{c_{1}}}(x)\right)^{2} \quad\left(\nu_{P 2}^{\bar{\tau}}(x)\right)^{2}, \sqrt{\left(\nu_{P 1}^{ \pm}\right.}$ $\left.\left.(x))^{2}+\left(\nu_{P 2}^{+}(x)\right)^{2}-\left(\nu_{P 1}^{ \pm}(x)\right)^{2}\left(\nu_{P 2}^{+}(x)\right)^{2}\right]\right)$

(3) $\lambda \widetilde{\alpha}=\left(\left[1-\left(1-\left(\mu_{\tilde{P}}^{-}(x)\right)^{2}\right)^{\lambda}, 1-\left(1-\left(\mu_{P}^{+}(x)\right)^{2}\right)^{\lambda}\right]\right.$, $\left.\left[\left(\nu_{\widetilde{P}}^{-}(x)\right)^{\lambda},\left(\nu_{P}^{+}(x)\right)^{\lambda}\right]\right)$

(4) $\widetilde{\alpha}^{\lambda}=\left(\left[\left(\mu_{P}^{-}(x)\right)^{\lambda},\left(\mu_{P}^{+}(x)\right)^{\lambda}\right],\left[1-\left(1-\left(\nu_{P}^{-}(x)\right)^{2}\right)^{\lambda}\right.\right.$, $\left.\left.1-\left(1-\left(\nu_{P}^{+}(x)\right)^{2}\right)^{\lambda}\right]\right)$

Definition 9 (see [13]). For two interval Pythagorean fuzzy numbers $\quad \widetilde{\alpha}_{i}=\left(\widetilde{P}\left(\mu_{\widetilde{P} i}^{-}(x), \mu_{\widetilde{P} i}^{+}(x)\right), \widetilde{P}\left(\nu_{\widetilde{P} i}^{-}(x), \nu_{\widetilde{P} i}^{+}(x)\right)\right)(i=$ $1,2)$, the Hamming distance $d\left(\widetilde{\alpha}_{1}, \widetilde{\alpha}_{2}\right)$ is defined as $d\left(\widetilde{\alpha}_{1}, \widetilde{\alpha}_{2}\right)=1 / 4 \quad\left(\left|\mu_{P 1}^{-}(x)-\mu_{P 2}^{-}(x)\right|+\left|\mu_{P 1}^{+}(x)-\mu_{P 2}^{+}(x)\right|+\right.$ $\left.\left|\nu_{\tilde{P}_{1}}^{-}(x)-\nu_{\tilde{P}_{2}}^{-}(x)\right|+\left|\nu_{P 1}^{ \pm}(x)-\nu_{P 2}^{ \pm}(x)\right|\right)$.
2.3. Weighted Average (WA) Operator Based on Interval Pythagorean Fuzzy Sets

Definition 10 (see [24]). There is a mapping WA: $R^{n} \longrightarrow R$, $\mathrm{WA}\left(x_{1}, x_{2}, x_{3}, \ldots, x_{n}\right)=\sum_{i=1}^{n} w_{i} x_{i}$, where $w=\left(w_{1}, w_{2}\right.$, $\left.w_{3}, \ldots, w_{n}\right)^{T}$ is the weighted vector of WA, satisfying $w_{i} \in[0,1]$ and $\sum_{i=1}^{n} w_{i}=1$. At this time, the function WA is called the weighted average operator.

Definition 11. For the interval Pythagorean fuzzy number $\widetilde{\alpha}_{i}=\left(\widetilde{P}\left(\mu_{\widetilde{P}}^{-}(x), \mu_{\mathbb{P}}^{+}(x)\right), \widetilde{P}\left(\nu_{\widetilde{P}}^{-}(x), \nu_{\widetilde{P}}^{+}(x)\right), \quad w_{i}(i=1,2,3, \ldots\right.$, $n)$ is the weight of the interval Pythagorean fuzzy number $\widetilde{\alpha}_{i}$, satisfying $w_{i} \in[0,1]$ and $\sum_{i=1}^{n} w_{i}=1$. The weighted average operator (IPVFWA) of the interval Pythagorean fuzzy number $\widetilde{\alpha}_{i}$ can be defined as follows:

$\operatorname{IVPFWA}\left(\widetilde{\alpha}_{1}, \widetilde{\alpha}_{2}, \widetilde{\alpha}_{3}, \ldots, \widetilde{\alpha}_{n}\right)=w_{1} \widetilde{\alpha}_{1} \oplus w_{2} \widetilde{\alpha}_{2} \oplus w_{3} \widetilde{\alpha}_{3} \oplus \cdots \oplus w_{4} \widetilde{\alpha}_{4}$

$$
=\left(\left[\sqrt{1-\prod_{i=1}^{n}\left(1-\left(\mu_{\widetilde{P} i}^{\bar{\tau}}(x)\right)^{2}\right)^{w_{i}}}, \sqrt{1-\prod_{i=1}^{n}\left(1-\left(\mu_{\widetilde{P}}^{ \pm}(x)\right)^{2}\right)^{w_{i}}}\right],\left[\prod_{i=1}^{n}\left(\nu_{P}^{\bar{P}}(x)\right)^{w_{i}}, \prod_{i=1}^{n}\left(\nu_{P}^{ \pm}(x)\right)^{w_{i}}\right]\right) .
$$

Theorem 1. If $\widetilde{\alpha}_{i}=\left(\widetilde{P}\left(\mu_{\widetilde{P}}^{-}(x), \mu_{\widetilde{p_{i}}}^{+}(x)\right), \widetilde{P}\left(\nu_{\widetilde{p_{i}}}^{-}(x), \nu_{\widetilde{P} i}^{+}(x)\right)\right)$ $(i=1,2,3, \ldots, n)$ is a group of interval Pythagorean ${ }^{P i}$ fuzzy numbers, then the IPVFWA operator is still an interval Pythagorean fuzzy number after integration.
Proof. Because $\widetilde{\alpha}_{i}=\left(\widetilde{P}\left(\mu_{\widetilde{P}_{i}}^{-}(x), \mu_{\widetilde{P} i}^{+}(x)\right), \widetilde{P}\left(\nu_{\widetilde{P}_{i}}^{-}(x), \nu_{\widetilde{P}^{+}}^{+}(x)\right)\right.$, according to Definition 8 ,

$$
\begin{aligned}
& w_{i} \widetilde{\alpha}_{i}=\left(\left[1-\left(1-\left(\mu_{\widetilde{P} i}^{\bar{\tau}}(x)\right)^{2}\right)^{w_{i}}, 1-\left(1-\left(\mu_{\widetilde{P i}^{+}}^{+}(x)\right)^{2}\right)^{w_{i}}\right],\left[\left(\nu_{\widetilde{P i}}^{-}(x)\right)^{w_{i}},\left(\nu_{\widetilde{P} i}^{+}(x)\right)^{w_{i}}\right]\right) \\
& \underset{i=1}{\oplus} w_{i} \widetilde{\alpha}_{i}=\stackrel{n}{\oplus}_{i=1}^{n}\left(\left[1-\left(1-\left(\mu_{\widetilde{P} i}^{\bar{\tau}}(x)\right)^{2}\right)^{w_{i}}, 1-\left(1-\left(\mu_{\widetilde{P i}}^{ \pm}(x)\right)^{2}\right)^{w_{i}}\right],\left[\left(\nu_{\widetilde{P} i}^{-}(x)\right)^{w_{i}},\left(\nu_{\widetilde{P} i}^{+}(x)\right)^{w_{i}}\right]\right) \\
& =\left(\left[\sqrt{1-\prod_{i=1}^{n}\left(1-\left(\mu_{\tilde{P i}}^{-}(x)\right)^{2}\right)^{w_{i}}}, \sqrt{1-\prod_{i=1}^{n}\left(1-\left(\mu_{\tilde{P i}}^{+}(x)\right)^{2}\right)^{w_{i}}}\right],\left[\prod_{i=1}^{n}\left(\nu_{\tilde{P}}^{-}(x)\right)^{w_{i}}, \prod_{i=1}^{n}\left(\nu_{\tilde{P}}^{+}(x)\right)^{w_{i}}\right]\right) \\
& =\operatorname{IVPFWA}\left(\widetilde{\alpha}_{1}, \widetilde{\alpha}_{2}, \widetilde{\alpha}_{3}, \ldots, \widetilde{\alpha}_{n}\right) \text {. }
\end{aligned}
$$

\section{Technique for Order Preference by Similarity to Ideal Solution (TOPSIS) Decision-Making Based on Interval Pythagorean Fuzzy Numbers}

This paper presents a new TOPSIS decision-making method based on interval Pythagorean fuzzy numbers.
Suppose there is a mixed multiattribute decision-making problem. The scheme set is $A=\left(A_{1}, A_{2}, A_{3}, \ldots, A_{n}\right)$, the attribute set is $B=\left(B_{1}, B_{2}, B_{3}, \ldots, B_{n}\right)$, and the decisionmaker set is $C=\left(C_{1}, C_{2}, C_{3}, \ldots, C_{n}\right)$. The evaluated value matrix given by the decision-maker $\mathrm{K}$ is

$$
D(k)=\left[\begin{array}{cccc}
\widetilde{\alpha}_{11} & \widetilde{\alpha}_{12} & \cdots & \widetilde{\alpha}_{1 n} \\
\widetilde{\alpha}_{21} & \widetilde{\alpha}_{22} & \cdots & \widetilde{\alpha}_{2 n} \\
\cdots & \cdots & \vdots & \cdots \\
\widetilde{\alpha}_{m 1} & \widetilde{\alpha}_{m 2} & \cdots & \widetilde{\alpha}_{m n}
\end{array}\right], \quad \widetilde{\alpha}_{i j}=\left(\mu_{\widetilde{P i j}}^{-}(x), \mu_{\widetilde{P i j}}^{+}(x), \nu_{\widetilde{P i j}}^{-}(x), \nu_{\widetilde{P i j}}^{+}(x)\right) .
$$


Step 1. The TOPSIS decision-making method is used to analyze interval Pythagorean fuzzy problems. First, the positive ideal matrix and negative ideal matrix of the interval Pythagorean fuzzy sets are defined, and multiple decision matrices are integrated into a comprehensive decision matrix.

According to the evaluated values of the all decisionmakers, the positive ideal matrix $L^{+}$and bilateral negative ideal matrices $L_{e}^{-}$and $L_{f}^{-}$are expressed as follows:

$$
\begin{aligned}
& L^{+}=\left[\begin{array}{cccc}
\widetilde{\alpha}_{11}^{+} & \widetilde{\alpha}_{12}^{+} & \cdots & \tilde{\alpha}_{1 n}^{+} \\
\tilde{\alpha}_{21}^{+} & \widetilde{\alpha}_{22}^{+} & \cdots & \tilde{\alpha}_{2 n}^{+} \\
\cdots & \cdots & \vdots & \cdots \\
\tilde{\alpha}_{m 1}^{+} & \tilde{\alpha}_{m 2}^{+} & \cdots & \tilde{\alpha}_{m n}^{+}
\end{array}\right], \\
& L_{e}^{-}=\left[\begin{array}{cccc}
\widetilde{\alpha}_{11}^{-e} & \widetilde{\alpha}_{12}^{-e} & \cdots & \widetilde{\alpha}_{1 n}^{-e} \\
\widetilde{\alpha}_{21}^{-e} & \widetilde{\alpha}_{22}^{-e} & \ldots & \widetilde{\alpha}_{2 n}^{-e} \\
\ldots & \ldots & \vdots & \ldots \\
\widetilde{\alpha}_{m 1}^{-e} & \widetilde{\alpha}_{m 2}^{-e} & \cdots & \widetilde{\alpha}_{m n}^{-e}
\end{array}\right] \text {, } \\
& L_{f}^{-}=\left[\begin{array}{cccc}
\widetilde{\alpha}_{11}^{-f} & \tilde{\alpha}_{12}^{-f} & \cdots & \widetilde{\alpha}_{1 n}^{-f} \\
\widetilde{\alpha}_{21}^{-f} & \widetilde{\alpha}_{22}^{-f} & \cdots & \widetilde{\alpha}_{2 n}^{-f} \\
\cdots & \cdots & \vdots & \cdots \\
\widetilde{\alpha}_{m 1}^{-f} & \widetilde{\alpha}_{m 2}^{-f} & \cdots & \widetilde{\alpha}_{m n}^{-f}
\end{array}\right] \text {, }
\end{aligned}
$$

where $\tilde{\alpha}_{i j}^{+}=\left(\sum_{k=1}^{C} \tilde{\alpha}_{i j}^{k} / C\right)$ is the mean of the evaluated values of all decision-makers, $\widetilde{\alpha}_{i j}^{-e}=\min \left[\widetilde{\alpha}_{i j}^{k}\right]$ is the minimum value below the average value, and $\tilde{\alpha}_{i j}^{-e}=\max \left(\tilde{\alpha}_{i j}^{k}\right)$ is the maximum value above the average.

Step 2. According to the positive and negative ideal matrices, the Hamming distance $d\left(\widetilde{\alpha}_{i j}^{k}, L\right)$ between each valuated value and the positive and negative ideal evaluated values can be obtained:

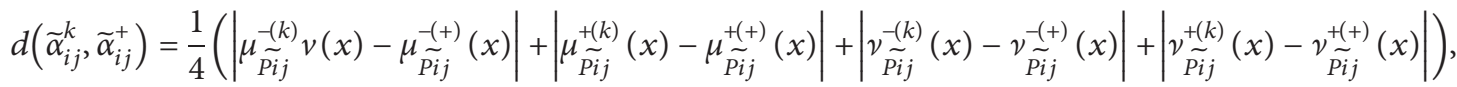

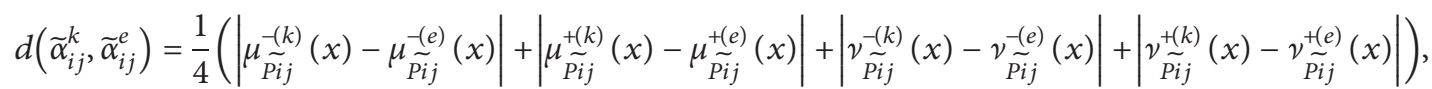

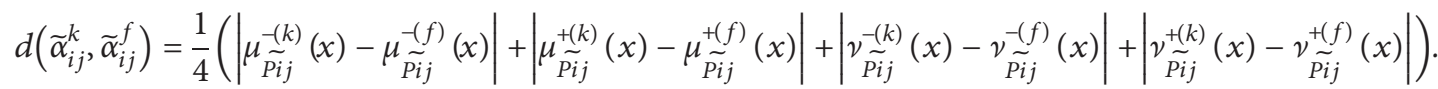

Step 3. The closeness of each evaluated value is calculated according to the valuated values of the positive and negative ideal matrices:

$$
r_{i j}^{k}=\frac{d\left(\tilde{\alpha}_{i j}^{k}, \tilde{\alpha}_{i j}^{e}\right)+d\left(\tilde{\alpha}_{i j}^{k}, \tilde{\alpha}_{i j}^{f}\right)}{d\left(\tilde{\alpha}_{i j}^{k}, \tilde{\alpha}_{i j}^{+}\right)+d\left(\tilde{\alpha}_{i j}^{k}, \tilde{\alpha}_{i j}^{e}\right)+d\left(\widetilde{\alpha}_{i j}^{k}, \tilde{\alpha}_{i j}^{f}\right)} .
$$

Step 4. The weights are determined as follows: $w_{i j}^{k}=\left(r_{i j}^{k} / \sum_{k=1}^{C} r_{i j}^{k}\right)$.

Step 5. The weighted average operator (IPVFWA) of the interval Pythagorean fuzzy number $\widetilde{\alpha}_{i}$ is mainly used to synthesize the decision matrix set of multiple decisionmakers into a single comprehensive decision evaluation matrix by obtaining the evaluated value weight of each decision-maker. Therefore, after determining the evaluated value weight of each decision-maker in the fourth step, the evaluation matrix set of each decision-maker can be synthesized into comprehensive decision matrices $M=\left(\widetilde{\alpha}_{i j}\right)_{m \times n}$ and $\underset{\alpha}{i j}=w_{i j}^{1} \widetilde{\alpha}_{i j}^{1} \oplus w_{i j}^{2} \widetilde{\alpha}_{i j}^{2} \oplus w_{i j}^{3} \widetilde{\alpha}_{i j}^{3} \oplus \cdots \oplus w_{i j}^{t} \widetilde{\alpha}_{i j}^{t}$ through the obtained weight and interval Pythagorean fuzzy number WA operator (IPVFWA).

Step 6. The positive and negative ideal solutions of the interval Pythagorean fuzzy comprehensive decision matrix $M=\left(\widetilde{\alpha}_{i j}\right)_{m \times n}$ and the Hamming distance between each evaluated value and the positive and negative ideal evaluated values are determined as follows:

Positive ideal solution: $L^{+}=\left(\widetilde{\alpha}_{1}^{+}, \widetilde{\alpha}_{2}^{+}, \widetilde{\alpha}_{3}^{+}, \ldots, \widetilde{\alpha}_{n}^{+}\right)$, where $\widetilde{\alpha}_{j}^{+}=\max \widetilde{\alpha}_{i j}$

Negative ideal solution: $L^{-}=\left(\widetilde{\alpha}_{1}^{-}, \widetilde{\alpha}_{2}^{-}, \widetilde{\alpha}_{3}^{-}, \ldots, \widetilde{\alpha}_{n}^{-}\right)$, where $\widetilde{\alpha}_{j}^{-}=\min \widetilde{\alpha}_{i j}$ 


\subsection{Hamming Distance}

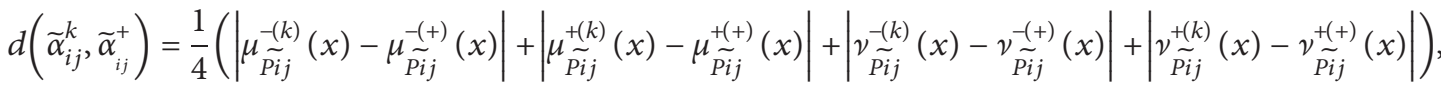

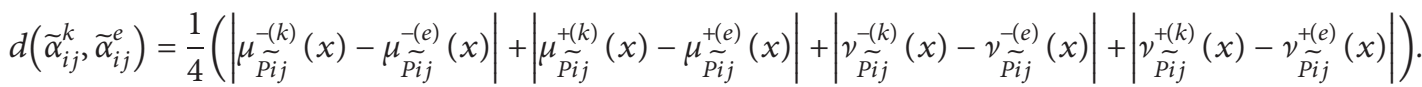

Step 7. The closeness coefficient is determined as follows:

$$
r_{i j}=\frac{d\left(\widetilde{\alpha}_{i j}, \tilde{\alpha}_{i j}^{-}\right)}{d\left(\widetilde{\alpha}_{i j}, \widetilde{\alpha}_{i j}^{+}\right)+d\left(\widetilde{\alpha}_{i j}, \widetilde{\alpha}_{i j}^{-}\right)} .
$$

Step 8. The greater the closeness coefficient of the scheme is, the closer the scheme is to being optimal.

\section{Example Analysis}

4.1. Evaluation Matrix. In this example, it is assumed that when an epidemic occurs in an area, there are three affected areas $\left(A=\left(A_{1}, A_{2}, A_{3}\right)\right.$ that need support and three experts evaluate the epidemic situation in an area. The attribute set of the evaluation includes the health status, epidemic prevention, and medical environment of the infected personnel in the affected area. The evaluation matrix of three decisionmakers is given in Table 1.

\subsection{Decision-Making Process}

Step 9. According to the evaluated values of all the decisionmakers, the positive ideal matrix $L^{+}$and bilateral negative ideal matrices $L_{e}^{-}$and $L_{f}^{-}$were found, as shown in Table 2.

Step 10. According to the positive and negative ideal matrices, the Hamming distance $d\left(\widetilde{\alpha}_{i j}^{k}, L\right)$ between each evaluated value and the positive and negative ideal evaluated values can be obtained, as shown in Table 3 .

Step 11. The closeness $r_{i j}^{k}$ of each evaluated value according to the evaluated values of the positive and negative ideal matrices were calculated, and the results are shown in Table 4.

Step 12. The weight $w_{i j}^{k}$ of each evaluated value was determined, and the results are shown in Table 5.

Step 13. At this time, the interval Pythagorean fuzzy number WA operator (IPVFWA) can be used to synthesize the evaluated value weight of each decision-maker obtained in Step 4, and the evaluation matrix sets of all of the decisionmakers are assembled into a comprehensive decision matrix $M=\left(\widetilde{\alpha}_{i j}\right)_{m \times n}$, as shown in Table 6.

Step 14. The positive and negative ideal solutions of the interval Pythagorean fuzzy comprehensive decision matrix, the Hamming distance between each evaluated value and the positive and negative ideal evaluated values, and the closeness degree and weight of each evaluated value were determined. The results are shown in Table 7.

Step 15. The weighted distance and closeness between the evaluated value of each scheme and the positive and negative ideal solutions were determined, and the results are shown in Table 8.

According to the results in Table 8 , scheme $A_{2}$ had the highest closeness and its scheme was the best. At the beginning of the decision-making process, each expert can be assigned a weight. However, weights were not given directly to the decision-makers in this example, but the Hamming distance between the evaluated value and the positive and negative ideal evaluated values was used to calculate the closeness of each evaluated value. The evaluated value weight of each decision-maker was determined through the closeness of the evaluated value, which ensured the objectivity of the whole decision-making process. At the same time, when the scheme score $(0.5135,0.6389,0.5301)$ was calculated using the interval Pythagorean fuzzy geometric weighted Bonferroni average operator of Jiang Yingying [25] in Step 6, the finite ranking of each scheme was still $(3,1,2)$ and the score results of each scheme were not much different. However, the TOPSIS decision-making method of the interval Pythagorean fuzzy numbers used in this paper show that the gap between the advantages and disadvantages of each scheme is very obvious, and as a result, it would be easier for decision-makers to make decisions.

Therefore, the TOPSIS hybrid multiattribute decisionmaking method based on interval Pythagorean fuzzy numbers has three advantages:

(1) In the TOPSIS hybrid multiattribute decisionmaking method based on interval Pythagorean fuzzy numbers, the decision-making method was used to provide the upper and lower limits of the membership and nonmembership and then decisions were made through the TOPSIS decision-making method. This method alleviates the loss of information in the decision-making process to a great extent, making the decision-making process more accurate and scientific.

(2) For mixed decision-making involving multiple decision-makers, when the decision-maker weight and attribute weight are unknown, the corresponding weight can be obtained from the decision matrix by using a TOPSIS hybrid multiattribute decision- 
TABLE 1: Evaluation matrix of three decision-makers.

\begin{tabular}{ccccc}
\hline & & $B_{1}$ & $B_{2}$ & $B_{3}$ \\
\hline & $A_{1}$ & $([0.7,0.8],[0.2,0.3])$ & $([0.7,0.8],[0.4,0.5])$ & $([0.4,0.6],[0.2,0.4])$ \\
$C_{1}$ & $A_{2}$ & $([0.5,0.6],[0.3,0.4])$ & $([0.5,0.6],[0.2,0.3])$ & $([0.4,0.5],[0.1,0.2])$ \\
& $A_{3}$ & $([0.6,0.8],[0.4,0.5])$ & $([0.7,0.9],[0.3,0.5])$ & $([0.6,0.7],[0.2,0.3])$ \\
\hline & $A_{1}$ & $([0.4,0.5],[0.2,0.3])$ & $([0.5,0.7],[0.3,0.4])$ & $([0.7,0.8],[0.4,0.5])$ \\
$C_{2}$ & $A_{2}$ & $([0.5,0.6],[0.4,0.5])$ & $([0.5,0.7],[0.4,0.5])$ & $([0.5,0.6],[0.2,0.3])$ \\
& $A_{3}$ & $([0.4,0.6],[0.1,0.3])$ & $([0.6,0.7],[0.4,0.5])$ & $([0.6,0.7],[0.2,0.4])$ \\
\hline & $A_{1}$ & $([0.5,0.6],[0.3,0.4])$ & $([0.7,0.8],[0.2,0.4])$ & $(0.7,0.8],[0.2,0.4])$ \\
$C_{3}$ & $A_{2}$ & $([0.4,0.5],[0.2,0.3])$ & $([0.6,0.8],[0.4,0.5])$ & $(0.4,0.5],[0.2,0.3])$ \\
& $A_{3}$ & $([0.7,0.8],[0.2,0.4])$ & & $([0.6,0.7],[0.4,0.5])$ \\
\hline
\end{tabular}

TABle 2: Positive and negative ideal matrices.

\begin{tabular}{ccccc}
\hline & & $B_{1}$ & $B_{2}$ & $B_{3}$ \\
\hline & $A_{1}$ & $([0.5333,0.6333],[0.2333,0.3333])$ & $([0.6000,0.7333],[0.3667,0.4667])$ & $([0.6000,0.7333],[0.2667,0.4333])$ \\
$L^{+}$ & $A_{2}$ & $([0.4667,0.5667],[0.3000,0.4000])$ & $([0.6000,0.7000],[0.2667,0.4000])$ & $([0.4333,0.5333],[0.1667,0.2667])$ \\
& $A_{3}$ & $([0.5667,0.7333],[0.2333,0.4000])$ & $([0.6000,0.8000],[0.3333,0.5000])$ & $([0.6000,0.7000],[0.2667,0.4000])$ \\
& $A_{1}$ & $([0.4,0.5],[0.3,0.4])$ & $([0.5,0.7],[0.4,0.5])$ & $([0.4,0.6],[0.4,0.5])$ \\
$L_{e}^{-}$ & $A_{2}$ & $([0.4,0.5],[0.4,0.5])$ & $([0.5,0.6],[0.4,0.5])$ & $([0.4,0.5],[0.2,0.3])$ \\
& $A_{3}$ & $([0.4,0.6],[0.4,0.5])$ & $([0.5,0.7],[0.4,0.5])$ & $([0.6,0.7],[0.4,0.5])$ \\
& $A_{1}$ & $([0.5,0.6],[0.2,0.3])$ & $([0.6,0.7],[0.3,0.4])$ & $([0.7,0.8],[0.2,0.4])$ \\
$L_{f}^{-}$ & $A_{2}$ & $([0.5,0.6],[0.2,0.3])$ & $([0.7,0.8],[0.2,0.4])$ & $([0.5,0.6],[0.2,0.3])$ \\
& $A_{3}$ & $([0.7,0.8],[0.1,0.3])$ & $([0.6,0.8],[0.3,0.5])$ & $([0.6,0.7],[0.2,0.4])$ \\
\hline
\end{tabular}

TABLE 3: Hamming distance $d\left(\tilde{\alpha}_{i j}^{k}, L\right)$ between each evaluated value and positive and negative ideal evaluated values.

\begin{tabular}{|c|c|c|c|c|c|}
\hline & & & $B_{1}$ & $B_{2}$ & $B_{3}$ \\
\hline \multirow{9}{*}{$C_{1}$} & \multirow{3}{*}{$d\left(\widetilde{\alpha}_{i j}^{k}, \tilde{\alpha}_{i j}^{+}\right)$} & $A_{1}$ & 0.1000 & 0.0583 & 0.1083 \\
\hline & & $A_{2}$ & 0.0167 & 0.0917 & 0.0500 \\
\hline & & $A_{3}$ & 0.0917 & 0.0583 & 0.0417 \\
\hline & \multirow{3}{*}{$d\left(\widetilde{\alpha}_{i j}^{k}, \widetilde{\alpha}_{i j}^{e}\right)$} & $A_{1}$ & 0.2000 & 0.0750 & 0.0750 \\
\hline & & $A_{2}$ & 0.1000 & 0.1000 & 0.0500 \\
\hline & & $A_{3}$ & 0.1000 & 0.1250 & 0.1000 \\
\hline & \multirow{3}{*}{$d\left(\widetilde{\alpha}_{i j}^{k}, \widetilde{\alpha}_{i j}^{f}\right)$} & $A_{1}$ & 0.1000 & 0.1000 & 0.1250 \\
\hline & & $A_{2}$ & 0.0500 & 0.1250 & 0.1000 \\
\hline & & $A_{3}$ & 0.1500 & 0.0500 & 0.0250 \\
\hline \multirow{9}{*}{$C_{2}$} & \multirow{3}{*}{$d\left(\widetilde{\alpha}_{i j}^{k}, \tilde{\alpha}_{i j}^{+}\right)$} & $A_{1}$ & 0.0833 & 0.0667 & 0.0917 \\
\hline & & $A_{2}$ & 0.0667 & 0.0583 & 0.0500 \\
\hline & & $A_{3}$ & 0.1333 & 0.0583 & 0.0167 \\
\hline & \multirow{3}{*}{$d\left(\widetilde{\alpha}_{i j}^{k}, \widetilde{\alpha}_{i j}^{e}\right)$} & $A_{1}$ & 0.0500 & 0.0500 & 0.1250 \\
\hline & & $A_{2}$ & 0.0500 & 0.0500 & 0.0500 \\
\hline & & $A_{3}$ & 0.1250 & 0.0250 & 0.0750 \\
\hline & \multirow{3}{*}{$d\left(\tilde{\alpha}_{i j}^{k}, \tilde{\alpha}_{i j}^{f}\right)$} & $A_{1}$ & 0.0500 & 0.0250 & 0.0750 \\
\hline & & $A_{2}$ & 0.1000 & 0.1250 & 0.0000 \\
\hline & & $A_{3}$ & 0.1250 & 0.0500 & 0.0000 \\
\hline \multirow{9}{*}{$C_{3}$} & \multirow{3}{*}{$d\left(\widetilde{\alpha}_{i j}^{k}, \widetilde{\alpha}_{i j}^{+}\right)$} & $A_{1}$ & 0.0500 & 0.0250 & 0.0667 \\
\hline & & $A_{2}$ & 0.0833 & 0.0667 & 0.0333 \\
\hline & & $A_{3}$ & 0.0583 & 0.0167 & 0.0583 \\
\hline & \multirow{3}{*}{$d\left(\widetilde{\alpha}_{i j}^{k}, \widetilde{\alpha}_{i j}^{e}\right)$} & $A_{1}$ & 0.0500 & 0.0250 & 0.2000 \\
\hline & & $A_{2}$ & 0.1000 & 0.1750 & 0.0000 \\
\hline & & $A_{3}$ & 0.2000 & 0.0500 & 0.0000 \\
\hline & \multirow{3}{*}{$d\left(\widetilde{\alpha}_{i j}^{k}, \widetilde{\alpha}_{i j}^{f}\right)$} & $A_{1}$ & 0.0500 & 0.0500 & 0.0000 \\
\hline & & $A_{2}$ & 0.0500 & 0.0000 & 0.0500 \\
\hline & & $A_{3}$ & 0.0500 & 0.0250 & 0.0750 \\
\hline
\end{tabular}

making method based on interval Pythagorean fuzzy numbers. Compared with the case where the weights of the decision-makers are known, this method can
TABLE 4: Closeness of each evaluated value.

\begin{tabular}{lllll}
\hline & & $B_{1}$ & $B_{2}$ & $B_{3}$ \\
\hline & $A_{1}$ & 0.7500 & 0.7500 & 0.6486 \\
$r_{i j}^{1}$ & $A_{2}$ & 0.9000 & 0.7105 & 0.7500 \\
& $A_{3}$ & 0.7317 & 0.7500 & 0.7500 \\
\hline \multirow{3}{*}{$r_{i j}^{2}$} & $A_{1}$ & 0.5455 & 0.5294 & 0.6857 \\
& $A_{2}$ & 0.6923 & 0.7500 & 0.5000 \\
& $A_{3}$ & 0.6522 & 0.5625 & 0.8182 \\
\hline \multirow{2}{*}{$r_{i j}^{3}$} & $A_{1}$ & 0.6667 & 0.7500 & 0.7500 \\
& $A_{2}$ & 0.6429 & 0.7241 & 0.6000 \\
& $A_{3}$ & 0.8108 & 0.8182 & 0.5625 \\
\hline
\end{tabular}

TABLE 5: Weight of each evaluated value.

\begin{tabular}{lllll}
\hline & & $B_{1}$ & $B_{2}$ & $B_{3}$ \\
\hline \multirow{3}{*}{$w_{i j}^{1}$} & $A_{1}$ & 0.3822 & 0.3696 & 0.3112 \\
& $A_{2}$ & 0.4027 & 0.3252 & 0.4054 \\
& $A_{3}$ & 0.3334 & 0.3520 & 0.3520 \\
$w_{i j}^{2}$ & $A_{1}$ & 0.2780 & 0.2609 & 0.3290 \\
& $A_{2}$ & 0.3097 & 0.3433 & 0.2703 \\
& $A_{3}$ & 0.2972 & 0.2640 & 0.3840 \\
$w_{i j}^{3}$ & $A_{1}$ & 0.3398 & 0.3696 & 0.3598 \\
& $A_{2}$ & 0.2876 & 0.3315 & 0.3243 \\
& $A_{3}$ & 0.3694 & 0.3840 & 0.2640 \\
\hline
\end{tabular}

effectively solve the problem of different types of attribute information and utilize the attribute advantages of each decision-maker to the greatest extent.

(3) Through the interval Pythagorean fuzzy TOPSIS hybrid multiattribute decision-making method, the 
TABle 6: Comprehensive evaluation matrix.

\begin{tabular}{lccc}
\hline & $B_{1}$ & $B_{2}$ & $B_{3}$ \\
\hline$A_{1}$ & $([0.5763,0.6806],[0.2295,0.3308])$ & $([0.6218,0.7427],[0.3711,0.4717])$ & $([0.6359,0.7546],[0.2512,0.4305])$ \\
$A_{2}$ & $([0.5618,0.5746],[0.2919,0.3946])$ & $([0.6124,0.7147],[0.2537,0.3933])$ & $([0.4305,0.5305],[0.1510,0.2545])$ \\
$A_{3}$ & $([0.7633,0.7569],[0.2051,0.3956])$ & $([0.6196,0.8275],[0.3350,0.5000])$ & $(0.6000,0.7000],[0.2402,0.3834])$ \\
\hline
\end{tabular}

TABLE 7: Positive and negative ideal matrices of the comprehensive matrix.

\begin{tabular}{lcccc}
\hline & & $B_{1}$ & $B_{2}$ & $B_{3}$ \\
\hline$L^{+}$ & & $([0.7633,0.7569],[0.2919,0.3956])$ & $([0.6218,0.8275],[0.3711,0.5000])$ & $([0.6359,0.7546],[0.2512,0.4305])$ \\
\hline$-\bar{L}$ & & $([0.5618,0.5746],[0.2051,0.3308])$ & $([0.6124,0.7147],[0.2537,0.3933])$ & $([0.4305,0.5305],[0.1510,0.2545])$ \\
\hline & $A_{1}$ & 0.0976 & 0.0283 & 0.0000 \\
$d\left(\widetilde{\alpha}_{i j}, \widetilde{\alpha}_{i j}^{+}\right)$ & $A_{2}$ & 0.0962 & 0.0866 & 0.1764 \\
& $A_{3}$ & 0.0217 & 0.0096 & 0.0371 \\
\hline & $A_{1}$ & 0.0362 & 0.0583 & 0.1764 \\
$d\left(\widetilde{\alpha}_{i j}, \widetilde{\alpha}_{i j}^{-}\right)$ & $A_{2}$ & 0.0376 & 0.0000 & 0.0000 \\
& $A_{3}$ & 0.1122 & 0.0770 & 0.1392 \\
\hline & $A_{1}$ & 0.2707 & 0.6734 & 1.0000 \\
$r_{i j}$ & $A_{2}$ & 0.2812 & 0.0000 & 0.0000 \\
& $A_{3}$ & 0.8379 & 0.8895 & 0.7894 \\
\hline & $A_{1}$ & 0.1392 & 0.3464 & 0.5144 \\
$w_{i j}$ & $A_{2}$ & 1.0000 & 0.0000 & 0.0000 \\
& $A_{3}$ & 0.3329 & 0.3534 & 0.3136 \\
\hline
\end{tabular}

TABLE 8: Weighted distance and closeness of the comprehensive matrix.

\begin{tabular}{lccc}
\hline & $A_{1}$ & $A_{2}$ & $A_{3}$ \\
\hline$w_{i j} d\left(\widetilde{\alpha}_{i j}, \widetilde{\alpha}_{i j}^{+}\right)$ & 0.0234 & 0.0962 & 0.0223 \\
$w_{i j} d\left(\widetilde{\alpha}_{i j}, \widetilde{\alpha}_{i j}^{-}\right)$ & 0.1160 & 0.0376 & 0.1082 \\
$r_{i j}$ & 0.1678 & 0.7188 & 0.1705 \\
Sort & 3 & 1 & 2 \\
\hline
\end{tabular}

decision matrices of multiple decision-makers are collected into a comprehensive evaluation matrix, and then, the TOPSIS decision-making method is used to solve the optimal scheme. In the whole decision-making process, the closeness values of each scheme calculated by the TOPSIS decisionmaking method twice would be significantly different. This would allow decision-makers to more easily select the optimal scheme, making the decision result more convincing.

\section{Conclusion}

In this paper, a mixed multiattribute decision-making problem was studied in which the decision-maker's weight and attribute weight are completely unknown and the attribute value is an interval Pythagorean fuzzy number. A TOPSIS mixed multiattribute decision-making method based on interval Pythagorean fuzzy numbers is proposed. The advantages of this method are as follows: First, in the decision-making process, by giving the upper and lower limits of the membership and nonmembership of the evaluated value, the information loss of decision-makers in the decision-making process is alleviated to the greatest extent. Second, the decision-makers are no longer given a decision weight directly in decision-making, fully utilizing the attribute advantages of each decision-maker and effectively solving the problems of different types of attribute information. Third, compared with other decision-making methods, the TOPSIS hybrid multiattribute decision-making method using interval Pythagorean fuzzy numbers increases the gap between the advantages and disadvantages of each scheme. As a result, the decision-making results are more convincing and it is easier for decision-makers to select the optimal scheme. The TOPSIS hybrid multiattribute decision-making method using interval Pythagorean fuzzy numbers not only reduces the subjectivity of the weight determination to a great extent but also uses interval Pythagorean fuzzy numbers to improve the risk impact of the deviation between membership and nonmembership on the decision-making results.

\section{Data Availability}

The data analyzed by the example in this paper are interval values randomly given in line with the assumptions in this paper. The data are real and can be used as a reference for readers.

\section{Conflicts of Interest}

The authors declare that they have no conflicts of interest.

\section{Acknowledgments}

This work was supported by the General Project of National Natural Science Foundation of China, "Price Distortion of 
Energy Factors: Causes, Measurement and Appropriate Correction Strategy" (71874073); the project supported by the National Social Science Foundation of China, "Research on Knowledge Transfer Game and Innovation Performance of Industry University Research Alliance" (19fglb029); and NSFC, "Research on Coupling Mechanism and Promotion Strategy of Knowledge Subject Collaborative Behavior and Value Creation in Innovation Ecosystem" (71771161).

\section{References}

[1] L. A. Zadeh, "Fuzzy sets," Information and Control, vol. 8, no. 3, pp. 338-353, 1965.

[2] L. A. Zadeh, "The concept of a linguistic variable and its application to approximate reasoning [D-J Series]," Information Sciences, vol. 8, no. 3, pp. 199-249, 1975.

[3] R. R. Yager, "Pythagorean fuzzy subsets ifsa world congress \& nafips meeting," 2013.

[4] X. Peng, "New similarity measure and distance measure for Pythagorean fuzzy set," Complex \& Intelligent Systems, vol. 5, no. 2, 2019.

[5] C. Ren, "Remoteness index-based Pythagorean fuzzy VIKOR methods with a generalized distance measure for multiple criteria decision analysis," Information Fusion, vol. 41, pp. 129-150, 2018.

[6] Peijia, Ren, and Zeshui, "Pythagorean fuzzy TODIM approach to multi-criteria decision making," Applied Soft Computing, vol. 42, pp. 246-259, 2016.

[7] H. Garg, "Generalized pythagorean fuzzy geometric aggregation operators using einstein t-norm and t-conorm for multicriteria decision-making process," International Journal of Intelligent Systems, vol. 32, no. 6, pp. 597-630, 2017.

[8] W. Liu, J. Chang, and X. He, "Generalized Pythagorean fuzzy integration operator and its decision application," Control and decision making, vol. 31, no. 12, pp. 2280-2286, 2016.

[9] Li Peng and Z. Shen, "Pythagorean Fuzzy Uncertain language decision method based on heronian average operator," China management science, vol. 29, no. 1, pp. 217-225, 2021.

[10] Li Peng, Z. Shen, S. Chen, and Q. Chai, "Pythagorean fuzzy group decision making method based on grey correlation method and ha operator [J]," Operations research and management, vol. 27, no. 10, pp. 56-62, 2018.

[11] X. Peng and Y. Yang, "Fundamental properties of intervalvalued pythagorean fuzzy aggregation operators[J]," International Journal of Intelligent Systems, vol. 31, no. 5, pp. 444-487, 2016.

[12] S. A. K. Muhammad, A. Ali, and S. Abdullah, "New extension of TOPSIS method based on Pythagorean hesitant fuzzy sets with incomplete weight information," Journal of Intelligent and Fuzzy Systems, vol. 35, no. 5, pp. 5435-5448, 2018.

[13] X. Zhang, "Multicriteria Pythagorean fuzzy decision analysis: a hierarchical QUALIFLEX approach with the closeness index-based ranking methods," Journal of Information Science, vol. 330, pp. 104-124, 2016.

[14] $\mathrm{Na} \mathrm{Li}, \mathrm{L}$. Gao, and L. Wang, "Interval Pythagorean fuzzy multi-attribute AQM decision-making method based on relative entropy and its application," Operations research and management, vol. 28, no. 1, pp. 79-85, 2019.

[15] Na Li, L. Gao, and L. Wang, "Interval pythagorean fuzzy interactive multi criteria decision model," Computer engineering and application, vol. 54, no. 22, pp. 246-251, 2018.

[16] W. Lin, M. Chen, L. Zhou, and Z. Lin, "An interval Pythagorean fuzzy VIKOR multi-attribute group decision- making method [J]," Operations research and management, vol. 30, no. 3, pp. 57-64, 2021.

[17] R. Umer, M. Touqeer, and A. H. Omar, "Selection of solar tracking system using extended topsis technique with interval type-2 pythagorean fuzzy numbers," Optimization and Engineering, vol. 34, 2021.

[18] S. Pishyar, H. Khosravi, and A. Tavili, "A combined AHP- and TOPSIS-based approach in the assessment of desertification disaster risk[J]," Environmental Modeling \& Assessment, vol. 25, no. 2, pp. 219-229, 2020.

[19] D. Kacprzak, "An extended TOPSIS method based on ordered fuzzy numbers for group decision making[J]," Artificial Intelligence Review, vol. 53, no. 3, pp. 2099-2129, 2020.

[20] G. Rezaei, P. Rani, and A. R. Mishra, "Extended pythagorean fuzzy TOPSIS method based on similarity measure for sustainable recycling partner selection," International Journal of Fuzzy Systems, vol. 20, pp. 1-33. In Press(In Press), 2020.

[21] Y. Du, W. Liu, and J. Chang, "Triangular Pythagorean fuzzy TOPSIS method based on attribute correlation," Practice and understanding of mathematics, vol. 51, no. 10, pp. 11-21, 2021.

[22] G. Qu, H. Zhang, Z. Liu, Z. Zhang, and Q. Zhang, "Based on Intuitionistic Fuzzy $\lambda$ - Multi attribute group decision making method of Shapley Choquet integral operator TOPSIS," System engineering theory and practice, vol. 36, no. 13, pp. 726-742, 2016.

[23] H. Zhao, Z. Xu, and M. Ni, "Hybrid fuzzy multiple attribute decision making," International Journal on Information, vol. 12, no. 5, pp. 1033-1044, 2009.

[24] R. R. Yager, "Generalized OWA aggregation operators," Fuzzy Optimization and Decision Making, vol. 3, no. 1, 2004.

[25] Y. Jiang, P. Duan, and L. Zhao, "Interval Pythagorean fuzzy geometric weighted Bonferroni average operator and its application," Journal of Qufu Normal University, vol. 47, no. 3, pp. 18-24, 2021. 